\title{
Syllis onkylochaeta sp. n., ein korallenfressender Polychaet (Syllidae) aus dem Korallenaquarium des Löbbecke-Museums
}

\author{
Gesa Hartmann-Schröder* \\ Biologische Anstalt Helgoland; Notkestraße 31, D-W-2000 Hamburg 52, \\ Bundesrepublik Deutschland \\ und \\ Zoologisches Institut und Zoologisches Museum der Universität Hamburg; \\ Martin-Luther-King-Platz 3, D-W-2000 Hamburg 13, Bundesrepublik Deutschland * *
}

\begin{abstract}
Syllis onkylochaeta sp.n. (Syllidae, Polychaeta), feeding on corals in the coral aquarium of the Löbbecke Museum. A new species of the genus Syllis is described feeding on a colony of corals of the genus Xenia (Xeniidae, Alcyonaria) in the coral aquarium of the Lobbecke Museum Düsseldorf. The origin of the corals is very probably Bali (Indonesia).
\end{abstract}

\section{EINLEITUNG}

Das vorliegende Material stammt aus dem Korallenaquarium des LöbbeckeMuseums in Düsseldorf. Dort leben die Polychaeten in enger Gemeinschaft mit einer Kolonie von Weichkorallen der Gattung Xenia (Xeniidae, Alcyonaria). Vermutlich fressen die Würmer an den Korallen, da deren Wachstum und Entwicklung - bereits geschwächt durch das Leben im Aquarium - gestört erscheinen. Nach Aussagen von R. Hebbinghaus haben sich die Polychaeten seit einem halben Jahr gut vermehrt. Die mir zur Verfügung gestellten Tiere sind geschlechtsreif; sie haben Stolone am Hinterkörper entwickelt, die Eier bzw. Spermien enthalten.

\section{BESCHREIBUNG DER ART}

Der Holotypus ist ein Weibchen mit 133 Borstensegmenten, von denen die hinteren 31 einem Stolon mit Eiern angehören. Länge etwa $45 \mathrm{~mm}$ (Tier leicht gekrümmt); größte Breite ohne Parapodien 1,75 mm. Färbung in Alkohol gelblich, der Stolon mit Eiern weißlich. Körper dorsal stärker aufgewölbt, so daß Parapodien in ventrolateraler Position. Körperoberfläche fein und unregelmäßig sekundär geringelt. Segmentgrenzen überall deutlich; vordere und hintere Segmente kurz, die mittleren etwas länger, bis zweimal so breit wie lang.

\footnotetext{
- Mitglied der Taxonomischen Arbeitsgruppe an der Biologischen Anstalt Helgoland

- Korrespondenzanschrift
} 
Prostomium fast vollständig unter das Peristomium eingezogen. Palpen ventrad umgebogen, breit, vollständig voneinander getrennt. Prostomium aus 2 Lappen bestehend, die vorn in der Mitte durch Einschnitt getrennt.

4 rötliche Augen, jederseits dicht beisammen, auf der Grenze zwischen 1. und 2. Segment durchschimmernd (Abb. 1A). Mediane Antenne hinter den lateralen etwa auf der Mitte des Prostomiums inserierend, an der Basis unregelmäßig, danach mehr oder weniger deutlich gegliedert, bis etwa zum 6. Borstensegment nach hinten reichend. Laterale Antennen am Vorderrand des Prostomiums, mit etwa 18 Gliedern und an der Basis undeutlich gerunzelt, etwas dünner als die mediane Antenne. Antennen und Cirren sich distad deutlich verschmälernd. Peristomium kragenartig über das Prostomium ragend, etwas kürzer als die folgenden Borstensegmente. Dorsale Tentakelcirren mit etwa 37 Gliedern, schwer zu zählen, etwa so lang wie die mediane Antenne. Ventrale Tentakelcirren mit etwa 14-15 Gliedern, nach hinten nicht weiter als bis zum Ende des 2. Borstensegments reichend. 1. Dorsalcirren etwa so lang wie die dorsalen Tentakelcirren. 2. Dorsalcirren etwas dünner und mit ca. 18 undeutlichen Gliedern viel kürzer. 3. Dorsalcirren mit über 40 undeutlichen Gliedern mindestens $2 \frac{1}{2} 2 \mathrm{mal}$ so lang wie die 2. Dorsalcirren. 4. Dorsalcirren anscheinend noch etwas länger (Glieder sehr undeutlich). Durch Fixierung Cirren möglicherweise etwas unterschiedlich gestreckt; sie sind insgesamt schlank und distal dünn, fast fadenförmig. In mittleren Segmenten Dorsalcirren etwas unregelmäßig länger und kürzer: die kürzeren etwas mehr als Segmentbreite lang, die längeren bis $2 \frac{1}{2} 2 \mathrm{mal}$ so lang. Im Hinterkörper Verhältnisse ähnlich, die kürzeren Dorsalcirren immer etwas dünner als die längeren.

Parapodien relativ kurz, abgestutzt, mit breit gerundeter prä- und wenig kürzerer, ähnlicher postsetalen Lamelle (Abb. 1B). Ventralcirren spindelförmig, mehr oder weniger deutlich gerunzelt, etwas weiter als der Borstenlappen vorragend. 5 Aciculae vorhanden: die meisten nadelförmig, eine davon manchmal etwas hakig gebogen; ihre Zahl auch im Hinterkörper nicht abnehmend (Abb. 1C). Sämtliche Parapodien mit zusammengesetzten und einfachen Borsten: im Vorderkörper 2 einfache und 3 zusammengesetzte (Abb. 1B), in mittleren Parapodien 2-3 einfache und 3 zusammengesetzte und hinten 1-2 einfache und 1 zusammengesetzte Borste. Die zusammengesetzten Borsten mit glattem Schaft und schlankem, glattem falcigeren Endglied, das in den meisten Fällen subdistal den Rest eines winzigen sekundären Zahns aufweist (Abb. 1D, E). In den allerletzten Segmenten sind Schaft und Endglied fast völlig miteinander verwachsen und zeigen deutlich die Entstehung der einfachen Borsten auf (Abb. 1F). Einfache Borsten meist etwas bis stark unterschiedlich in der Größe pro Parapod: vorn leicht, nach hinten zu stärker hakig gebogen und mit kleinem subdistalen Höcker unterhalb des konkaven Abschnitts (Abb. 1D-F).

Pharynx relativ breit, hell, bis Ende des 6 . Borstensegments reichend; mit 12 weichen Papillen am Eingang; Zahn etwas hinter dem Eingang im 2. Borstensegment liegend. Magen vom 7.-14. Borstensegment, mit 31 Muskelzellreihen.

Stolon: 1. Segment mit kleinen Parapodien und Dorsalcirren; keine weiteren Anhänge, auch Augen nicht zu erkennen. Prall gefüllt mit kleinen Eiern.

Lebende Paratypen: 9 : Hauptabschnitt (Ammentier) mit 147 Borstensegmenten, dahinter Stolon mit 39 Borstensegmenten und Eiern in der Leibeshöhle. Im Auflicht Vorderkörper durchsichtig grünlich; ab etwa 50. Borstensegment zunehmend undurchsichtig und weißlich; Eier weiß. Hellbraune Pigmentbänder am Vorderrand der 


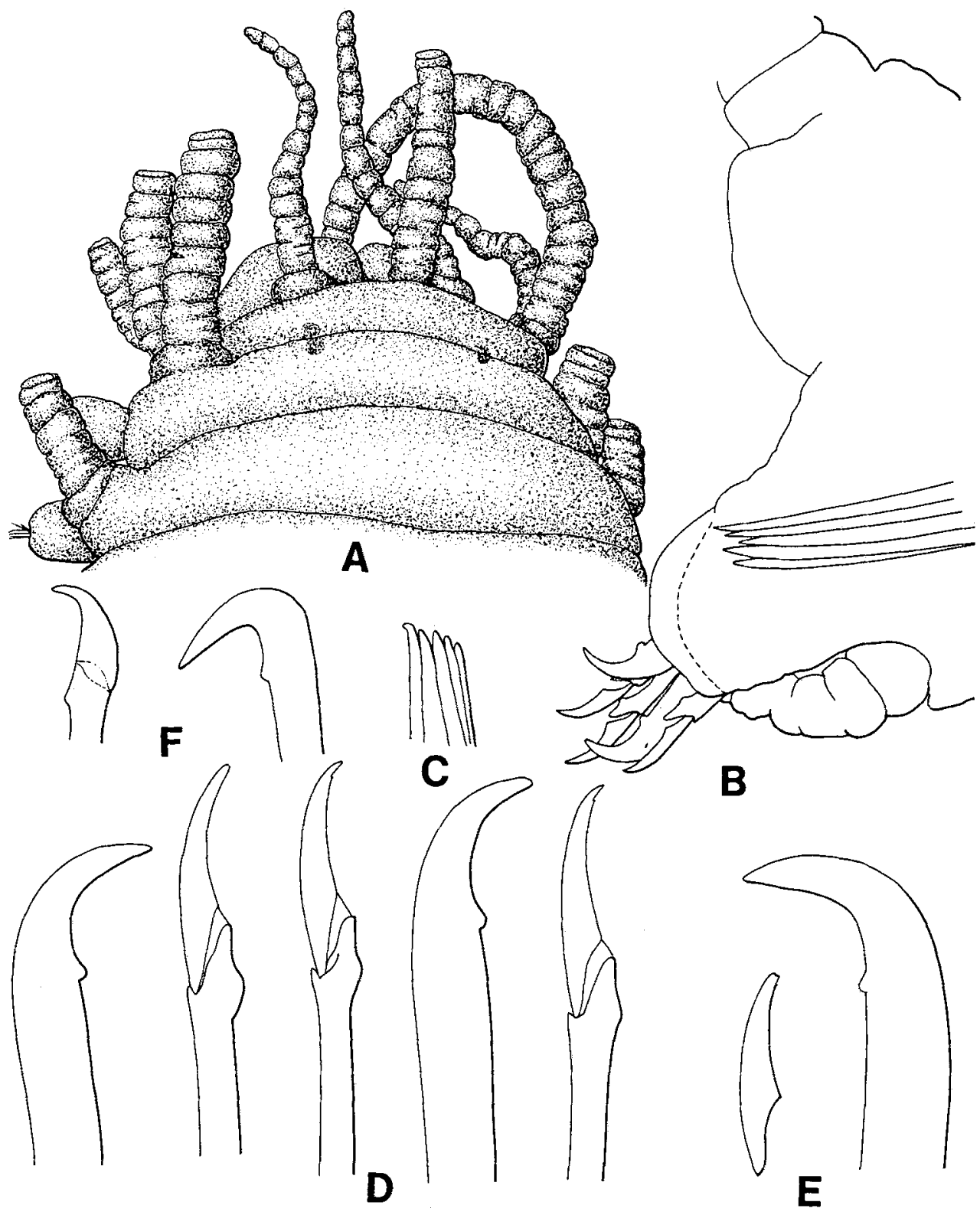

Abb. 1. Syllis onkylochaeta sp.n. A: Vorderende von dorsal. B: 9. Parapod von vorn. C: Spitzen der Aciculae. D: Borsten des 9. Parapods (von oben nach unten). E: Endglied einer zusammengesetzten Borste und einfache Borste aus 70. Parapod. F: "Zusammengesetzte" und einfache Borste aus hinterem Parapod 
Segmente, auf dem Stolon auch verstreut braune Pigmentkörnchen über das ganze Segment. 1. Segment des Stolons mit 4 kleinen roten Augen, kleinen Parapodien und Dorsalcirren; keine weiteren Anhänge wie beim Holotypus.

o: : Ammentier mit 99, Stolon mit 35 Borstensegmenten. Vorderkörper weißlichgelblich, durchsichtig. Stolon mit Spermien. 1. Segment des Stolons mit 4 roten Augen, 2 kleinen Anhängen am Vorderrand jederseits, 1 mehr dorsal, 1 mehr ventral (Antennen, Palpen) und dahinter ein kleines Parapod mit Dorsalcirrus.

3. Paratypus hinten unvollständig, mit 40 Borstensegmenten; weißlich bis sehr schwach grünlich.

Länge der lebenden Tiere nicht meßbar, da die Tiere in ständiger Bewegung und dabei sich verknäulend, jedoch deutlich länger als der fixierte Holotypus. Prostomium frei vom Peristomium, mit 2 deutlichen seitlichen Lappen, zwischen denen die mediane Antenne entspringt. Augen braun, sich nicht jederseits berührend, im Trapez angeordnet. Mediane Antenne etwa $21 / 2 \mathrm{mal}$ so lang wie Prostomium und Palpen zusammen; laterale Antenne etwa $2 / 3$ so lang wie die mediane; beide sehr undeutlich geringelt. Palpen bis doppelt so lang wie das Prostomium, meist ventrad umgebogen und seitlich etwas komprimiert. Peristomium etwas lappen- oder kragenartig über den Hinterrand des Prostomiums ragend und die hinteren Augen bedeckend. Dorsale Tentakelcirren etwa so lang wie die mediane Antenne, die ventralen wie die lateralen Antennen. Dorsalcirren glatt erscheinend, gleichmäßig in der Länge alternierend: die längeren so lang wie doppelte Segmentbreite, die kürzeren etwa 3/4 so lang; die längeren nach oben, die kürzeren etwas nach unten gerichtet und etwas dünner. Borsten meist nur wenig vorgestreckt wie beim fixierten Holotypus, nur beim Kriechen weiter vorragend. Pharynx und Magen wie beim Holotypus.

Locus typicus: Korallenaquarium des Löbbecke-Museums Düsseldorf; an Xenia sp. (Xeniidae, Alcyonaria); 18. 01. 1990: Holotypus (ZMH: P-20222). Gleicher Fundort; 21. 06. 1990: 3 Paratypen (ZMH: P-20223).

\section{DISKUSSION}

Einfache Borsten - abgesehen von den beiden einfachen oberen und unteren im Borstenbündel der Typosyllis-Arten und Verwandten - gibt es bei den Sylliden nur in den Gattungen Haplosyllis Langerhans, Syllis Savigny, Geminosyllis Imajima und Trypanosyllis Claparède (in der Untergattung Trepanobia). Geminosyllis und Trypanosyllis scheiden bei der Zuordnung der oben beschriebenen Art aus, da sie neben dem SyllisZahn im Pharynx einen Trepan entwickelt haben. Arten der Gattung Haplosyllis fehlen die zusammengesetzten Borsten: es sind nur einfache Borsten vorhanden. Damit bleibt nur die Zuordnung in die Gattung Syllis.

Bei ihr sind die einfachen Borsten - entstanden durch Verlust des Endgliedes oder durch Verschmelzen von Schaft und Endglied der zusammengesetzten Borsten - meist auf die mittlere Körperregion beschränkt, wie z. B. die Y-förmigen einfachen Borsten von Syllis gracilis Grube. Bei der vorliegenden Art sind einfache und zusammengesetzte Borsten vom 1. Parapod an vorhanden, und die zusammengesetzte Borste der hinteren Parapodien zeigt Verwachsungserscheinungen zwischen Schaft und Endglied, damit die Entstehung der einfachen Borsten demonstrierend.

Ähnlich stark hakige einfache Borsten sind in der Gattung Syllis nur bei Syllis 
lycochaetus Grube anzutreffen. Weder Grube (1878) noch Ehlers (1920), der als einziger die Art wiedergefunden hat, geben jedoch Auskunft über die Verteilung der einfachen Borsten. Grube schreibt, daß viele Borsten abgebrochen waren und daß er nur (meist 3) einfache Borsten vorgefunden habe, während Ehlers auch zusammengesetzte Borsten neben den einfachen gesehen hat. Demnach könnte die Borstenverteilung - einfache und zusammengesetzte - ähnlich sein wie bei der oben beschriebenen Art. Die einfachen Borsten sind nach Grube stark hakig gekrümmt (seine Fig. 2c), zeigen aber keinen Höcker subdistal auf der konkaven Seite wie bei $S$. onkylochaeta sp. n. Daneben beschreibt und bildet er ab eine lange einfache, nadelförmige Borste. Möglich ist, daß die Nadel der Schaft einer zusammengesetzten Borste ist, dessen Endglied abgefallen ist, und daß Grube den kleinen Höcker an den großen hakenförmigen Borsten übersehen hat - bei der obigen Art sieht man ihn auch nur beim abpräparierten Parapod unter dem Mikroskop. Unterschiedlich scheinen aber doch mindestens die zusammengesetzten Borsten zu sein: Nach Ehlers sind die Endglieder bei $S$. lycochaetus ebenso hakig gekrümmt wie die einfachen Borsten, was für die obige Art nicht zutrifft. Aber auch die von Grube und Ehlers erwähnten paarigen Höcker auf den Segmenten sind bei der neuen Art nicht vorhanden. Übereinstimmend sind dagegen die undeutlich bzw. teilweise langgegliederten Dorsalcirren und die ventrolaterale Position der Parapodien. Insgesamt betrachtet kann eine Identität beider Arten nicht völlig ausgeschlossen werden. Auf Grund der genannten Unterschiede halte ich die obige Art jedoch für eine neue Art. Typenmaterial von Syllis lycochaetus scheint nicht (mehr) zu existieren. Die Grubeschen Polychaeten von den Philippinen (leg. Semper) sind im Museum für Naturgeschichte in Breslau (Wroclaw) deponiert; S. lycochaetus fehlt jedoch im Typenkatalog.

Danksagung. Herzlich bedanken möchte sich die Autorin bei G. Reinicke, Institut für Hydrobiologie der Universität Essen, für die Überlassung eines Exemplares eines Sylliden aus dem Korallenaquarium des Löbbecke-Museums. Dieses Tier ist der Holotypus der neuen Art. Außerdem sei Dr. $\mathrm{H}$. Bosch, Löbbecke-Museum Düsseldorf, gedankt, der mir freundlicherweise noch lebende Tiere (die 3 Paratypen) zukommen ließ. Dank gebührt auch R. Hebbinghaus, ebenfalls Löbbecke-Museum, für seine Mühen, die Tiere aus dem Aquarium zu fangen.

\section{LITERATUR}

Ehlers, E., 1920. Polychaeten von Java und Amboina. Ein Beitrag zur Kenntnis der malaiischen Strandfauna. - Abh. K. Ges. Wiss. Göttingen $10(7), 1-73$.

Grube, A. E., 1878. Annulata Semperiana. Beiträge zur Kenntnis der Annelidenfauna der Philippinen nach den von Herrn Prof. Semper mitgebrachten Sammlungen. - Mém. Acad. Sci. St. Petersburg 25, 1-300. 